\title{
Adult Presentation of Diastematomyelia with Terminal Epidermoid Cyst Arising from One Hemicord: A Case Report
}

\author{
Hatim Belfquih, MD*, Brahim El Mostarchid, MD, Hassan Baallal, MD, Amine Adraoui, \\ $M D$ and Ali Akhaddar, MD
}

Department of Neurosurgery, Avicenne Military Hospital, Cadi Ayyad University, Marrakech, Université Mohammed V de Rabat, Morocco

\section{Background}

Diastematomyelia, or split cord malformations (SCM) are congenital spinal anomalies with incomplete splitting of the spinal cord in the sagittal plane. It is usually seen in children, and presentation in adulthood is extremely rare.

To report on an adult patient with diastematomyelia and discuss the embryological basis and related developmental sequelae of this split-cord malformation.

\section{Case Presentation}

A 34-year-old female patient presented with a 4-month history of bilateraly lombosciatalgy and left lower-extremity paresthesia, Urinary urgency began within the last month, although bowel function was normal. She had a normal neurologic examination. Plain radiographs showed fusion of L2 and L3 vertebral bodies, with a bony septum at L2-L3 revealed clearly by tomodensitometry. Magnetic resonance imaging (MRI) revealed two hemicords in the spinal canal, separating at the level of D9, an intradural extramedullary mass, arising from teminal of one hemicords at the L3-L4 level, mostly consistent with an epidermoid tumor, the conus was low lying, ending at L3 (Figure 1). A midline lumbar exposure was followed by L2-L4 laminectomy, The mass was grossly consistent with epidermoid tumor; this was debulked, the septum divided the spinal cord was resected. The patient grew without a neuro-logical deterioration during nine postoperative months.

\section{Discussion}

Diastematomyelia or SCMs are a spinal dysraphism characterized by the presence of an osseous, cartilaginous, or fibrous septum that splits the spinal cord into two distinct hemicords. They can be further classified as to whether each hemicord is contained within its own dural sac (type I) as opposed to containment of both hemicords within a single common dural sheath (type II).

SCMs are often located in the lumbar and dorsolumbar regions. Sinha, et al. reported incidence rates for cervical, cervicodorsal, dorsal, dorsolumbar, lumbar and lumbosacral locations as $3 \%, 1 \%, 13 \%, 28 \%, 50 \%$ and $5 \%$, respectively. In most type I SCM patients, the osseocartilaginous septum extend from the vertebral body to the neural arch, where it unites with the bone on the opposite side [1]. These types of septum are termed "complete", as in our case. However, in some patients, the septum may be incomplete.

SCM may be accompanied by many congenital spinal disorders such as low-lying cord, hydroxylian, lipoma meningomyelocele. Symptoms may range from back pain to progressive neurologic deterioration in both children and adults. In addition to the presence of scoliosis, cutaneous lesions, including capillary hemangioma, subcutaneous lipoma, hypertrichosis, and dermal sinus, are often markers of diastematomyelia [2].

MRI is a highly effective technique for determining SCM and its accompanying anomalies. An osseocartilaginous septum can be detected on all axial images. A fibrous septum, however, can only be detected on high-resolution T2-weighted images. Tomodensitometry is able to display the bony septum well. In addition, on coronal and sagittalreformatted images, any accompanying vertebral anomalies may be better demonstrated with the new multidetector CT scanning technology [2].

*Corresponding author: Hatim Belfquih, MD, Department of Neurosurgery, Avicenne Military Hospital, Cadi Ayyad University, Marrakech, Université Mohammed V de Rabat, 40000, Morocco

Accepted: November 27, 2021

Published online: November 29, 2021

Citation: Belfquih H, El Mostarchid B, Baallal H, et al. (2021) Adult Presentation of Diastematomyelia with Terminal Epidermoid Cyst Arising from One Hemicord: A Case Report. J Neurosurg Res Rev 4(1):70-71 


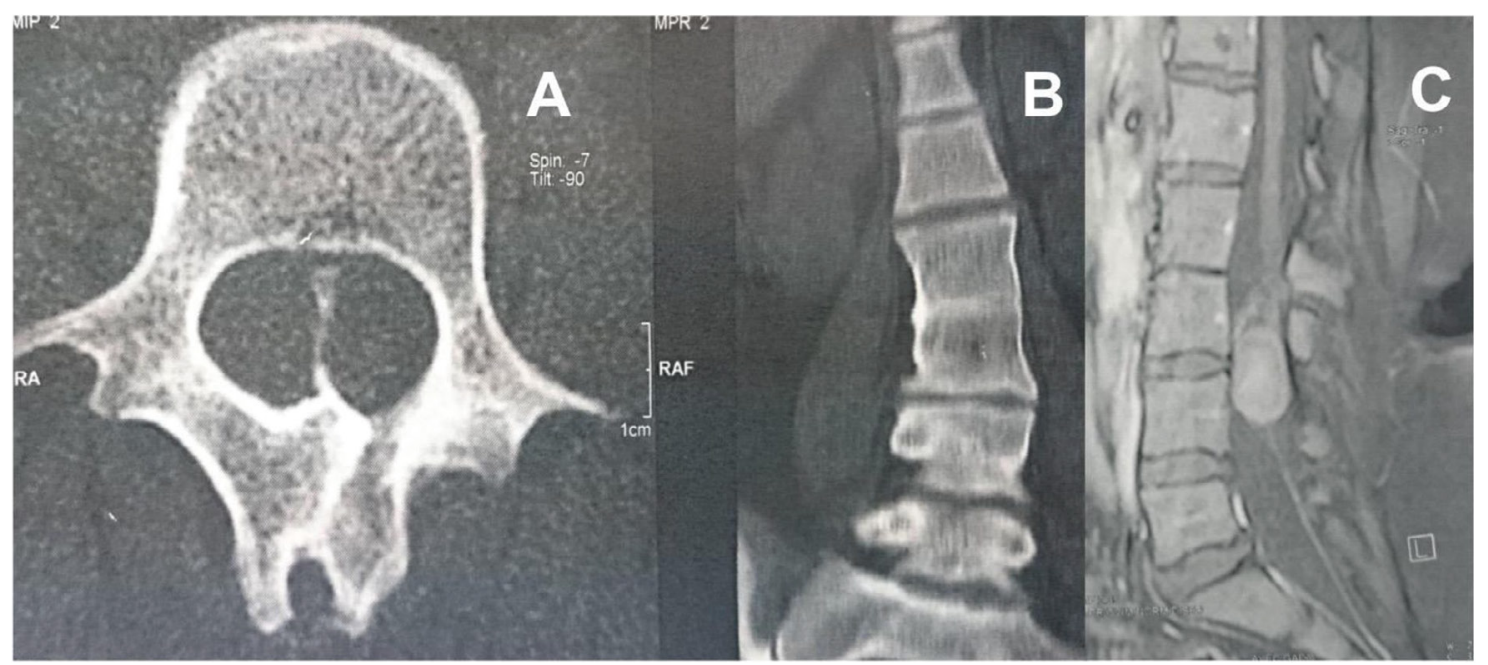

Figure 1: Tomodensitometry showing fusion of $L 2$ and $L 3$ vertebral bodies, with the bony septum separating the two hemicords (A, B). Magnetic resonance imaging (MRI) revealed two hemicords in the spinal canal, separating at the level of D9, An intradural extramedullary mass, arising from teminal of one hemicord at the L3-L4 level (C).

Multiple cases report diastematomyelia with a concurrent lipomatous tethered cord and embryonic cyst within the vertebral canal; few are associated with mass lesions such as an epidermoid tumor [3]. Indeed, only three previous reports noted diastematomyelia with accompanying intradural extramedullary epidermoid tumor.

In adults, surgery for diastematomyelia is indicated when stenosis or cord tethering causes neurologic deficits. With the exception of scoliosis, prophylactic spur removal is not recommended in asymptomatic adults. For complete bony spur removal, a wide dural opening and duraplasty are often required [4].

\section{Conflicts of Interest}

The author has no conflicts of interest to declare.

\section{References}

1. Porensky P, Muro K, Ganju A (2007) Adult presentation of spinal dysraphism tandem diastematomyelia. Spine J 7: 622-626.

2. Ozturk E, Sonmez G, Mutlu H, et al. (2008) Split-cord malformation and accompanying anomalies. J Neuroradiol 35: 150-156.

3. Lewandrowski KU, Rachlin JR, Glazer PA (2004) Diastematomyelia presenting as progressive weakness in an adult after spinal fusion for adolescent idiopathic scoliosis. Spine J 4: 116-119.

4. Patankar T, Krishnan A, Armao D, et al. (2000) Diastematomyelia and epidermoid cyst of the hemicord. AJR Am J Roentgenol 174: 1793-1794.

DOI: $10.36959 / 363 / 419$ 